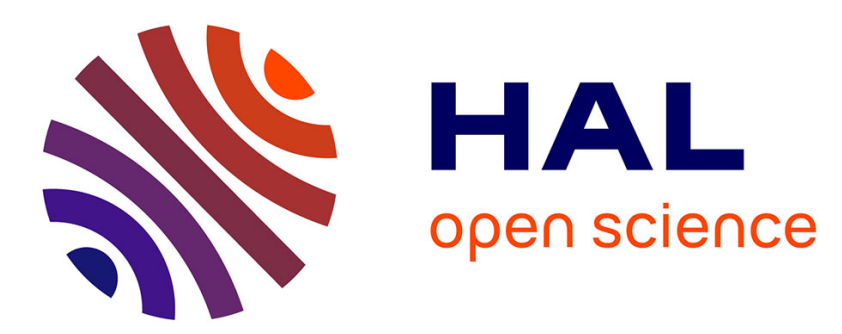

\title{
Gouvernance d'entreprise et performances sectorielles: une réévaluation de la fiabilité des scores et des mesures de bonne gouvernance
}

Jackie Krafft, Yiping Qu, Jacques-Laurent Ravix

\section{To cite this version:}

Jackie Krafft, Yiping Qu, Jacques-Laurent Ravix. Gouvernance d'entreprise et performances sectorielles: une réévaluation de la fiabilité des scores et des mesures de bonne gouvernance. Economie et Prévision, 2011, 197-198 (1/2), pp.145-158. hal-00493371

\section{HAL Id: hal-00493371 \\ https://hal.science/hal-00493371}

Submitted on 18 Jun 2010

HAL is a multi-disciplinary open access archive for the deposit and dissemination of scientific research documents, whether they are published or not. The documents may come from teaching and research institutions in France or abroad, or from public or private research centers.
L'archive ouverte pluridisciplinaire HAL, est destinée au dépôt et à la diffusion de documents scientifiques de niveau recherche, publiés ou non, émanant des établissements d'enseignement et de recherche français ou étrangers, des laboratoires publics ou privés. 


\section{Titre :}

Gouvernance d'entreprise et performances sectorielles: Une réévaluation de la fiabilité des scores et des mesures de bonne gouvernance ${ }^{1}$

\section{Noms, affiliations et adresses e-mail :}

Jackie Krafft * ; Yiping Qu ** ; Jacques-Laurent Ravix ***

$*, * *, * * *$ Université de Nice-Sophia Antipolis, CNRS-GREDEG.

E-mail : Jackie.Krafft@gredeg.cnrs.fr

E-mail : Qu.Yiping@gredeg.cnrs.fr

E-mail : $\underline{\text { ravix@gredeg.cnrs.fr }}$

\section{Résumé long :}

Depuis les années 1980, la littérature sur la gouvernance d'entreprise s'est développée sur deux fronts. D’une part, elle s'est attachée à améliorer la compréhension scientifique du modèle de gouvernance d'entreprise et, d'autre part, elle a tenté d'élaborer des scores et des mesures fiables de "bonne gouvernance », c'est-à-dire les meilleures pratiques de contrôle des dirigeants par les investisseurs tendant à garantir efficacité et performance des firmes. Ces indicateurs de bonne gouvernance ont été régulièrement critiqués dans la période plus récente. Quatre ensembles de critiques peuvent être évoqués. Premièrement, on invoque souvent l'idée que l'élaboration des scores et des mesures ne traduit pas les développements analytiques les plus récents, et donc que les critères fournissent une évaluation obsolète et partielle, voire fausse, conduisant à des sur-ou sous-estimations de la situation réelle des entreprises en matière de gouvernance. Deuxièmement, on reproche aussi aux indicateurs de bonne gouvernance d'être fondés sur un seul critère d'évaluation, par exemple la présence dans les conseils d'administration d'une majorité de membres extérieurs, alors qu'une mesure fiable requerrait des dimensions multicritères (composition des comités d'audit et de rémunération, politiques en matière de protection contre les OPA, durée et caractéristiques du mandat des dirigeants, etc.). Troisièmement, les indicateurs de bonne gouvernance sont suspectés d'être marqués par un biais court-termiste, qui déprécierait injustement les firmes et les industries engagées dans des stratégies d'innovation de plus long terme. Quatrièmement, enfin, on estime que ces critères donnent un reflet illusoire de la réalité des modes de gouvernance puisque, même dans les sociétés où les scores sont bons, on peut observer des manquements importants, tant de la part des dirigeants qui échappent encore au contrôle des actionnaires et des investisseurs, que de la part des actionnaires et investisseurs 
qui n'assument pas leur fonction première qui est la prise de risque dans leurs avances aux activités industrielles.

Le présent article s'insère dans le débat sur la nécessité de disposer de scores et de mesures fiables de la gouvernance d'entreprise. Notre contribution s'appuie principalement sur le constat de la quasi-absence de référence aux effets sectoriels dans l'élaboration des critères existants. L'article montre que les reproches dont font l'objet ces indicateurs peuvent être mieux fondés si l'on prend en compte explicitement les disparités sectorielles qui, contrairement à ce que l'on peut lire dans la littérature, permettent de mieux interpréter les variations de scores de bonne gouvernance, et leur fiabilité. Cet article se propose de se focaliser sur l'analyse empirique de ces questions, en considérant qu'il est illusoire de définir un modèle de gouvernance de manière normative, mais en proposant au contraire une voie de recherche positive sur la gouvernance.

\section{Codes JEL :}

G30

\section{Mots clefs :}

Gouvernance d'entreprise, maximisation de la valeur actionnariale, classements et standards internationaux de gouvernance d'entreprise, performance des firmes, industries innovatrices versus industries traditionnelles 
Depuis les années 1980, la littérature sur la gouvernance d'entreprise s'est développée sur deux fronts. D'une part, elle s'est attachée à améliorer la compréhension scientifique du modèle de gouvernance d'entreprise et, d'autre part, elle a tenté d'élaborer des scores et des mesures fiables de «bonne gouvernance », c'est-à-dire les meilleures pratiques de contrôle des dirigeants par les investisseurs tendant à garantir efficacité et performance des firmes. Les fondements analytiques de la théorie de l'agence, couplés à des instruments traditionnels de théorie financière, permettent de définir de nouveaux indicateurs servant d'outil d'évaluation pour le praticien et orientant les politiques économiques en la matière. A titre d'exemple, on peut noter, d'une part, la mobilisation des grands cabinets de consultants tels que Stern Stewart, Boston Consulting Group ou McKinsey qui proposent des mesures de l'impact de la gouvernance d'entreprise sur les valeurs de marché et, d'autre part, le développement plus récent de bases de données commerciales à destination des investisseurs telles que RiskMetrics / International Shareholder Services (RM/ISS), The Corporate Library (TCL), ou Governance Metrics International(GMI).

Ces indicateurs de bonne gouvernance ont été régulièrement critiqués dans la période plus récente. Quatre ensembles de critiques peuvent être mentionnés. Premièrement, on invoque souvent l'idée que l'élaboration des scores et des mesures ne traduit pas les développements analytiques les plus récents, et donc que les critères fournissent une évaluation obsolète et partielle, voire fausse, conduisant à des sur- ou sous-estimations de la situation réelle des entreprises en matière de gouvernance. Deuxièmement, on reproche aussi aux indicateurs de bonne gouvernance d'être fondés sur un seul critère d'évaluation, par exemple la présence dans les conseils d'administration d'une majorité de membres extérieurs, alors qu'une mesure fiable requerrait des dimensions multicritères (composition des comités d'audit et de rémunération, politiques en matière de protection contre les OPA, durée et caractéristiques du mandat des dirigeants, etc.). Troisièmement, les indicateurs de bonne gouvernance sont suspectés d'être marqués par un biais court-termiste, qui déprécierait injustement les firmes et les industries engagées dans des stratégies d'innovation de plus long terme. Quatrièmement, enfin, on estime que ces critères donnent un reflet illusoire de la réalité des modes de gouvernance puisque, même dans les sociétés où les scores sont bons, on peut observer des manquements importants, tant de la part des dirigeants qui échappent encore au contrôle des actionnaires et des investisseurs, que de la part des actionnaires et investisseurs qui n'assument pas leur fonction première qui est la prise de risque dans leurs avances aux activités industrielles. 
Le présent article a pour objet de contribuer au débat sur l'exigence croissante de disposer de scores et de mesures fiables de la gouvernance d'entreprise en réalisant un bilan critique des critères existants. Notre critique s'appuie principalement sur le constat de la quasi-absence de référence aux effets sectoriels dans la littérature. Il s'agira donc de montrer que les reproches dont font l'objet les indicateurs existants peuvent être mieux fondés si l'on prend en compte explicitement les disparités sectorielles. Celles-ci permettent en effet de mieux interpréter les variations de scores de bonne gouvernance, et leur fiabilité. Cet article se propose de se focaliser sur l'analyse empirique de ces questions, en considérant qu'il est illusoire de définir un modèle de gouvernance de manière normative, mais en proposant au contraire une voie de recherche positive sur la gouvernance.

L'article se structure comme suit. La première partie fournit une revue de la littérature qui traduit les fondements analytiques de la gouvernance d'entreprise en critères opérationnels et la généralisation de l'utilisation de ces critères et leur codification au sein de bases de données aidant à la décision des investisseurs et des politiques. Au terme de cette section, nous identifions deux propositions de recherche concernant la pertinence de ces critères : 1) l'évaluation de la gouvernance d'entreprise, qu'elle soit appréhendée par les critères simples fournis par les cabinets de consultants ou par des analyses multicritères adoptées plus récemment, échoue généralement à fournir une mesure claire des performances des entreprises ; 2) les différences sectorielles sont fondamentales pour juger de la fiabilité des scores et des mesures. Ces disparités sectorielles font que l'adoption des mesures de bonne gouvernance a un impact différencié sur la volatilité des performances. La deuxième partie de l'article présente les données sur lesquelles nous testons ces deux propositions. Ces données proviennent de la base RM/ISS, qui est de loin la plus complète et la plus fréquemment utilisée dans le milieu académique et dans le monde des affaires. La troisième partie présente les résultats de l'étude empirique des deux propositions énoncées. Ces résultats montrent que la fiabilité des scores et des mesures dépend de leur capacité à intégrer un grand nombre de critères, et que, parmi ces critères, l'appartenance sectorielle est décisive. La quatrième partie offre une discussion des résultats et une proposition pour des recherches futures.

\section{La littérature empirique sur la gouvernance d'entreprise}


Depuis son origine, la question de la gouvernance d'entreprise est directement liée à la recherche de l'efficacité de l'entreprise. Chez Berle et Means (1932), la séparation entre propriété et contrôle est fondatrice du conflit d'intérêt entre dirigeants et actionnaires qui engendre de l'inefficience dans les grandes entreprises. Les premières contributions de la théorie de l'agence (Jensen et Meckling, 1976 ; Fama, 1980 ; Fama et Jensen, 1983) ont également pour objet de montrer que les asymétries d'information entre un principal, l'actionnaire ou l'investisseur, et un agent, le dirigeant d'entreprise, seront réduites par la définition d'un contrat optimal conduisant l'agent à toujours chois ir des actions dans l'intérêt du principal, et à limiter les comportements discrétionnaires. La prise en compte explicite des situations de rationalité faible et de coûts d'écriture et de négociation des contrats par les approches en termes de coûts de transaction (Williamson, 1988) ou de droits de propriété (Hart, 1995) conduisent également à proposer différents mécanismes de contrôle à mettre en place par les actionnaires ou investisseurs afin de résoudre les problèmes d'opportunisme et de discipline des dirigeants. Pour une revue récente et complète de la recherche sur la gouvernance d'entreprise, nous renvoyons le lecteur au numéro spécial préparé par Bebchuk et Weisbach (2009).

La prise en compte de l'impact de la gouvernance d'entreprise sur la performance sur les marchés, et non plus seulement sur des gains d'efficacité interne, apparaît avec l'émergence de la théorie de la valeur actionnariale qui codifie la relation entre actionnaires et dirigeants (Jensen, 1986). Ainsi, au tout début des années 1990, un certain nombre de cabinets de consultants tentent de traduire les principes de la théorie de l'agence en termes d'indicateurs opérationnels. C'est alors qu'apparaissent les mesures de l'impact de la bonne gouvernance sur la valeur des actions de l'entreprise, telles que Shareholder Value Criteria de McKinsey, Total Share Return / Total Business Return du Boston Consulting Group, ou Economic Value Added / Market Value Added de Stern Stewart. Des classements à destination des investisseurs sont alors développés en fonction de la manière dont les entreprises répondent à ces critères, et sont largement repris dans les rapports annuels d'activité des entreprises et médiatisés dans la presse spécialisée du monde des affaires (tel que le magazine Fortune, par exemple). Ces classements, fondés à l'origine sur l'observation de l'impact du mode de gouvernance d'entreprise sur les performances de marchés des entreprises américaines, tendent à s'instaurer comme norme et à se diffuser largement internationalement ( $c f$. par exemple, les Principes de Gouvernance publiés régulièrement par l'OCDE). Cette période 
marque le début de l'évaluation de la gouvernance d'entreprise monocritère, fondée sur l'impact sur les valeurs boursières.

Le milieu des années 1990 fait apparaître de nouveaux éclaircissements théoriques qui vont engendrer une explosion des travaux empiriques. A titre d'exemple, le nombre de publications académiques référencées en économie sur le thème est multiplié par trois entre les années 1980 et 1990, allant de 2000 à 6000 (source : JSTOR) ${ }^{2}$. Schleifer et Vishny (1997) identifient les principaux mécanismes qui permettent aux «pourvoyeurs de ressources financières aux entreprises » de s'assurer un «retour sur leurs investissements ». Ces mécanismes sont de deux types : internes (surveillance du conseil d'administration, procédure interne de contrôle et audit interne, structure de pouvoir, rémunérations et compensations) et externes (fusionsacquisitions, OPA, système réglementaire et légal, concurrence et régulation, marché du travail des dirigeants). Cette évaluation, toujours orientée vers la valeur actionnariale, se fonde désormais sur différents déterminants quantifiables et marque donc l'avènement d'une mesure multicritère de la gouvernance d'entreprise.

Parallèlement, les premiers travaux empiriques menés à partir de la fin des années 1990 continuent de mesurer l'impact d'un déterminant pris isolément sur la performance de l'entreprise. Le décalage entre les travaux théoriques et empiriques peut s'expliquer par la difficulté d'accéder à des données complètes sur l'ensemble des dimensions de gouvernance d'entreprise. Il s'ensuit que les résultats obtenus dans les différentes contributions demeurent souvent peu cohérents, voire contradictoires. A titre d'exemple, Hermalin et Weisbach (2003) montrent, dans un article fondateur, que la prédominance de membres extérieurs dans les conseils d'administration augmente la performance boursière, alors que Bhagat, Black et Blair (2004) avancent au contraire que les firmes où prédominent les relations de long terme avec les investisseurs (relational investors) sont celles qui ont les meilleurs résultats. En ce qui concerne les rémunérations et les compensations des dirigeants, il est encore moins sûr que les faire dépendre des résultats de l'entreprise soit dans la pratique un système efficace, si l'on en veut pour preuve un certain nombre de scandales récents, et certaines analyses de ces scandales (Lie, 2005).

Ces résultats contradictoires s'expliquent sans doute par le fait que la recherche empirique sur la gouvernance d'entreprise est relativement récente. Le stade de maturité auquel doit arriver un programme de recherche nouveau, qui correspond à l'identification de grandes tendances 
empiriques robustes soutenant des hypothèses partagées de manière consensuelle par la communauté scientifique, n'est pas encore atteint. Toutefois, il faut aussi admettre que des travaux menés sur des périodes différentes, dans des pays différents, sur des populations de firmes différentes, et mesurant des déterminants qui eux-mêmes ont différentes composantes, ne peuvent aisément conduire à des résultats immédiatement lisibles.

L'émergence de réels travaux multicritères sur la gouvernance est un phénomène très récent qui coïncide avec l'élaboration de bases de données exclusivement dédiées à cette question (Gompers et al., 2003 ; Core et al., 2006). Plusieurs bases de données ont ainsi été développées au cours de ces dernières années : RiskMetrics / International Shareholder Services (RM/ISS), The Corporate Library (TCL), ou Governance Metrics International (GMI). Leur point commun est d'évaluer de manière systématique les forces, faiblesses et caractéristiques des pratiques de gouvernance des firmes par rapport au standard que représente la maximisation de la valeur actionnariale. L'adoption de ce standard au niveau d'une firme, d'une industrie ou d'un pays est calculée sur la base d'un indice multicritère qui intègre les différentes dimensions de la gouvernance. Les bases de données offrent donc une collecte d'information la plus exhaustive possible sur ces différentes dimensions et, pour chaque dimension, un système de scores (sommables en scores plus globaux) propre à chaque fournisseur de base de données. Le tableau 1 ci-dessous présente les principales dimensions qui sont prises en compte dans ces bases et comment celles-ci sont scorées par rapport à la norme de gouvernance d'entreprise par la maximisation de la valeur actionnariale (GEMVA, dans le tableau 1). Par exemple, si l'on s'intéresse à la dimension «conseil », et plus particulièrement à la composition du Conseil d'Administration (CA), on pourra dire que celleci est conforme aux principes de bonne gouvernance lorsque le CA est composé d'une large majorité de membres extérieurs, et le score sera donc de 5 (sur une échelle de 0 à 5 ). Si en revanche le CA est composé uniquement de membres internes à l'entreprise, alors les mesures de bonne gouvernance ne sont pas respectées et le score sera de 0. Ces sous-scores sont ensuite sommés pour donner un score par grande catégorie, i.e. «conseils », «audits », «charte/statuts », «compensation des dirigeants et des cadres», et «pratiques progressistes », qui donnent enfin un score global. Pour une firme, ce score global est comparé aux performances de toutes les autres firmes de la base, également évaluées sur les mêmes indices boursiers (en général MSCI, NYSE, ou S\&P 500). 
La fiabilité de ces scores reste encore très discutée dans la littérature, et notamment sur le fait qu'il demeure difficile de synthétiser dans un seul indicateur final la complexité des modes de gouvernance dans le monde concret, ou plus généralement d'accorder une confiance totale à ces indicateurs et à leurs évaluateurs (Ertugrul et Hedge, 2009 ; Koehn et Ueng, 2005 ; Bebchuk et Hamdani, 2009 ; Dasmodaran, 2005 ; Sonnenfeld, 2004 ; Froud, Haslam, Johal, Williams, 2000).

On voit ainsi apparaître des arguments selon lesquels l'obtention d'un bon (respectivement d'un mauvais) score n'est pas forcément le reflet indiscutable d'une bonne (respectivement d'une mauvaise) performance. Dans cette perspective, on insiste sur le fait que le score qu'Enron ou d'autres compagnies frauduleuses dégageaient juste avant leur chute était souvent excellent dans la plupart des bases de données. De même, Google apparaît en général comme une entreprise mal notée, du fait de la spécificité de sa structure de propriété et de décision plus proche de la jeune pousse que de la grande société par action.

Compte tenu de ces limites, ce qui se dégage des travaux récents, c'est une approche de la gouvernance plus positive, abandonnant l'idée qu'une norme systématique s'applique en la matière. Ainsi on étudie plutôt les conditions de cohérence qui amènent l'entreprise, l'industrie, ou le pays, à recevoir un tel score. Il existe en effet une diversité persistante des modes de gouvernance d'entreprise qui résiste à la convergence totale vers le modèle de maximisation de la valeur actionnariale, impliquant de mauvais scores sans pour autant traduire une réelle inefficience. Cette diversité s'exprime fortement au niveau des pays et des firmes; preuve en est l'abondante littérature sur ces deux thèmes (Allen, 2005 ; Becht, Jenkinson, et Mayer, 2005 ; Coffee, 2005 ; Aglietta et Rebérioux, 2005 ; Denis et McConnell, 2003 ; Grandori, 2004 ; Zingales, 2000 ; Hansmann, 1996 ; Blair, 1995, Aoki, 1984) dans laquelle on trouve en particulier le développement d'autres perspectives de gouvernance que celle de la maximisation de la valeur actionnariale, telles que la gouvernance par la valeur partenariale ou la gouvernance par la valeur critique des ressources. En revanche, la prise en compte de spécificités sectorielles est plus rarement mise en évidence. En effet, à part les travaux qui soulignent l'aspect court-termiste de la gouvernance par la maximisation de la valeur actionnariale et ses effets perturbateurs sur les firmes innovatrices (Fransman, 2004 ; 
Lazonick, 2007 ; Krafft et Ravix, 2005 ; Krafft, Qu, et Ravix, 2008), peu de travaux mettent en avant de manière systématique des effets sectoriels.

En conclusion de cette revue de littérature, nous pouvons noter que les évaluations disponibles en matière de gouvernance d'entreprise présentent deux caractéristiques. Primo, elles se situent sur un axe opposant l'unicité et la diversité des critères retenus. Secundo, les évaluations contradictoires paraissent refléter l'absence d'une dimension fondamentale, celle du secteur d'appartenance des entreprises. Ces conclusions nous conduisent à élaborer deux propositions de recherche :

Proposition 1 : L'évaluation monocritère de la gouvernance d'entreprise ne peut aller dans le sens d'une amélioration de la fiabilité des scores et mesures. Pour autant, l'évaluation multicritères reste souvent difficile à interpréter de manière strictement objective.

Proposition 2 : Les différences sectorielles sont fondamentales pour juger de la fiabilité des scores et des mesures. Ces disparités sectorielles font que l'adoption des mesures de "bonne gouvernance » a un impact différencié sur la volatilité des performances.

Dans la suite de ce travail, nous montrons que ces deux propositions peuvent être analysées à partir d'une étude empirique fondée sur une base de données de référence. Cette étude empirique nous permet, d'une part, de répondre à l'exigence croissante de disposer de scores et de mesures fiables sur la gouvernance d'entreprise et, d'autre part, de montrer que l'exigence de fiabilité peut être mieux fondée si l'on prend en compte explicitement des disparités sectorielles.

\section{Les bases de données disponibles en matiè re de gouvernance d'entreprise}

Depuis une dizaine d'années, plusieurs bases de données concurrentes se sont créées pour évaluer la gouvernance d'entreprise. Notre objectif n'est pas de réaliser une présentation exhaustive de ces bases, mais plutôt de décrire les caractéristiques des bases de données les plus utilisées dans le milieu académique et dans le monde des affaires, et de justifier notre choix d'utiliser une de ces bases. 
The Corporate Library (TCL) (www.thecorporatelibrary.com) a été créée en 1999. Son objectif est de fournir à ses clients investisseurs ou chercheurs des informations brutes, mais aussi des analyses plus fines sur la gouvernance d'entreprise, notamment sur les rémunérations et compensations du PDG et des dirigeants. La base de données regroupe 3200 entreprises américaines et canadiennes. Depuis 2003, cette firme propose également des classements sur l'efficacité des conseils d'administration (appelés TCL Ratings) qui regroupent quatre éléments principaux : la structure du CA, les compensations du PDG, les mesures anti-OPA, et les gains des dirigeants. Ces éléments sont chacun affectés de la pondération suivante : $50 \%, 30 \%, 10 \%$ et $10 \%$. Les classements se font par lettre (A, B, C, D et $\mathrm{F}$ ) et établissent une hiérarchie, depuis les entreprises qui ont le $\mathrm{CA}$ le plus efficace jusqu'à celles qui ont le CA le moins efficace. TCL fournit également des sous-classements prenant en compte d'autres aspects de la gouvernance, tels que la composition du CA, la capacité des actionnaires à intervenir dans les décisions, les problèmes de régulation et de contentieux, etc.

Governance Metrics International (GMI) (www.gmiratings.com) est légèrement plus récente, puisqu'elle a été fondée en 2000 dans le but de donner la possibilité à des clients de suivre la politique des firmes en matière de gouvernance d'entreprise. Elle regroupe des informations sur 4200 entreprises du monde entier. Les scores de GMI intègrent également différents aspects de la gouvernance, tels que l'obligation du CA à rendre des comptes aux actionnaires, la transparence des informations notamment financières, les droits des actionnaires, les compensations, la structure de propriété, le marché du contrôle et le comportement de la firme. GMI propose un score croissant de 1 à 10. Pour chaque firme, les scores sont comparés à l'ensemble des scores attribués aux firmes répertoriées dans GMI. Il est possible aussi d'avoir une comparaison du score de la firme avec le score des firmes de son pays d'origine.

Nous avons choisi pour notre étude empirique la base de données RiskMetrics / International Shareholder Services (RM/ISS) (www.isscgq.com). C'est la plus ancienne puisque ISS a été créée en 1985, et c'est également la plus complète puisque des données opérationnelles et systématiques sur la gouvernance sont disponibles depuis 2001 pour les entreprises américaines et 2003 pour les autres entreprises (Europe, Asie, etc.). Le score de RM/ISS est appelé Corporate Governance Quotient (CGQ), et il est fondé sur des informations journalières sur 7500 compagnies au total, dont environ 2500 en Europe. Le CGQ peut être calculé au niveau de la firme (une firme qui a un score CGQ de 80\% fait mieux en termes de gouvernance d'entreprises que $80 \%$ de toutes les autres firmes de la base listées dans l'indice 
boursier MCSI EAEFE), au niveau de l'industrie (25 industries sont couvertes par la base et si une industrie a un score de $80 \%$, cela veut dire qu'elle fait mieux en termes de gouvernance d'entreprise que $80 \%$ des autres industries), ou au niveau des pays (si un pays a un score de $80 \%$, alors il a de meilleures performances en termes de gouvernance que $80 \%$ des autres pays).

\section{Etude empirique des deux propositions}

Pour l'étude de la première proposition, nous avons utilisé l'ensemble des données dont nous disposions, c'est-à-dire les scores journaliers collectés du 29 octobre 2003, date de la création de la base de donnée RM/ISS pour l'Europe, jusqu'au 31 mai 2007, date de fin de notre abonnement à la base. Le nombre de firmes par année qui est utilisé pour cette étude est de 1781 pour 2003, 1697 pour 2004, 2270 pour 2005, 2381 pour 2006, et 2358 pour 2007. Pour éclairer de manière simple les enjeux de l'évaluation monocritère versus multicritère, il suffit de se focaliser sur la première grande dimension des mesures de bonne gouvernance présentée dans le tableau 1 ci-dessus : la dimension «conseils $»^{3}$. Cette dimension a un score total qui se décompose en 10 sous-scores. L'annexe 1 regroupe l'ensemble des résultats récoltés dans la base de données sur les sous-dimensions : composition du CA, composition du comité de gouvernance, composition du comité de compensation, taille du CA, changements dans la taille du CA, votes cumulatifs, participation du PDG à d'autres CA, participation des dirigeants à d'autres $\mathrm{CA}$, participation des anciens PDG au CA, et séparation Président/Directeur Général. Pour chaque sous-dimension, nous avons indiqué si les mesures de gouvernance correspondent aux standards de gouvernance d'entreprise par la maximisation de la valeur actionnariale (GEMVA). Le résultat auquel nous aboutissons est que GEMVA n'est la pratique dominante dans la population des firmes que pour la moitié des sousdimensions considérées. On note, par exemple, que si l'on retient les principales sousdimensions de la GEMVA identifiées dans les travaux pionniers dans ce domaine (cf., par exemple, Hermalin et Weisbach, 2003), telles que la composition du CA, la composition du comité de gouvernance, la composition de comité de compensation, alors on est amené à dire que la GEMVA n'est globalement pas adoptée dans la population de firmes étudiées. Si l'on prend en compte les autres sous-dimensions, on voit alors que cette conclusion est erronée, puisque 5 sous-dimensions indiquent que GEMVA est dominante. Cela confirme bien (Proposition 1) que l'évaluation monocritère n'est pas satisfaisante, car elle peut donner une vision partielle et donc biaisée de la réalité de l'adoption du standard de GEMVA. Pour 
autant, l'évaluation multicritères dans le cas de la dimension «conseil » (mais qui concerne aussi les autres dimensions du tableau 1) reste difficile à interpréter puisque 5 dimensions vont dans le sens de l'adoption de la GEMVA, alors que les 5 autres indiquent que la GEMVA n'est pas adoptée de manière dominante.

Pour analyser la seconde proposition et faire apparaître les disparités sectorielles, nous avons réduit la base de données de manière à identifier les effets de l'adoption de la GEMVA dans deux secteurs aux caractéristiques a priori distinctes: un secteur traditionnel (Agroalimentaire $^{4}$ ) et un secteur technologique (Technologie de l'information et de la communication $^{5}$ ). Pour ces deux secteurs nous avons cherché à mesurer l'impact de l'adoption de la GEMVA sur les performances boursières. Nous avons utilisé les données sur la même période que précédemment ; le nombre de firmes est plus faible du fait de la prise en compte de deux secteurs mais le nombre élevé d'observations nous permet d'avoir des résultats de qualité.

Le tableau 2 présente des statistiques descriptives sur l'adoption de la GEMVA dans chaque secteur exprimée en log (LnGEMVA), et l'évolution du cours boursier exprimée en log également $(\operatorname{LnSP})$.

INSERER TABLEAU 2

Il apparait que l'industrie des TIC a une meilleure adoption moyenne du standard de GEMVA que l'industrie agro-alimentaire mais, dans le même temps, de moindres performances boursières. Cela contredit le standard GEMVA dont l'adoption doit normalement accroître la performance.

Pour comprendre cette contradiction, nous posons l'équation suivante qui permet de saisir l'impact d'une variation d'un score mesurant la bonne gouvernance sur les performances boursières. Les variables de contrôle sont MV, la valeur de marché (ou taille), et DY, les dividendes, également exprimés en log.

$$
\operatorname{LnSP}_{i, t}=\alpha+\beta_{1} \operatorname{LnGEMVA}_{i, t}+\beta_{2} \operatorname{LnMV}_{i, t}+\beta_{3} \operatorname{LnDY}_{i, t}+v_{i, t}
$$


Le modèle utilisé est un modèle à effet fixe : les grandeurs observées en termes de variables explicatives sont considérées comme non aléatoires. Le tableau 3 résume les résultats.

\section{INSERER TABLEAU 3}

Le tableau montre qu'il existe une relation positive et significative entre les de ux variables, à la fois dans l'industrie agro-alimentaire et dans l'industrie des TIC. Toutefois, l'impact d'un changement de score (à la hausse comme à la baisse) est bien plus fort dans les TIC que dans l'agro-alimentaire. Il y a donc bien des spécificités sectorielles à prendre en compte dans l'élaboration et dans l'interprétation des scores de bonne gouvernance (Proposition 2). Ces différences sectorielles font que l'adoption des mesures de bonne gouvernance a un impact plus fort sur la volatilité des performances dans les industries technologiques que dans les industries traditionnelles.

\section{Discussion et conclusion}

A l'issue de cette analyse, deux éléments de discussion peuvent être mis en évidence. Le premier est lié à la distinction entre analyses monocritère et multicritères ; il a trait à la fonction normative de la gouvernance d'entreprise et au rôle que peut jouer la recherche académique sur l'interprétation des bonnes pratiques de gouvernance. Le second point est un retour de la logique industrielle, révélée par la mise en œuvre de la dimension sectorielle de la gouvernance d'entreprise.

Pour ce qui est de la relation entre la règle normative et la recherche positive en matière de gouvernance d'entreprise, nous avons vu dans un premier temps que les bonnes pratiques étaient promues sur la base d'indicateurs simples élaborés par des cabinets de consultants. L'évaluation monocritère était en effet adaptée à l'activité de conseil, et à la diffusion de règles de bonnes pratiques par des organismes tels que l'OCDE. C'est dans un second temps que l'intervention de la recherche académique a conduit à critiquer ces critères simples pour proposer des évaluations plus élaborées. Des bases de données se sont alors créées pour fournir des recueils d'informations plus systématiques et complets sur la question. Toutefois, les résultats que nous obtenons dans cet article montrent que les efforts réalisés sont toujours critiquables du point de vue du chercheur. En effet, le score ne reste que peu informatif si l'on 
n'a pas accès à ses composantes et, en définitive, ne se distingue pas vraiment de l'indice simple. Il reste élaboré pour orienter la décision des investisseurs, et son caractère trop synthétique peut facilement conduire à des erreurs de choix tout comme dans le cas des indices simples. En revanche, disposer des sous-scores va dans le sens de l'identification d'une diversité de modes de gouvernance d'entreprise que le chercheur doit interpréter.

Dans cette perspective d'interprétation, on peut montrer que les hypothèses sous jacentes aux modèles de best practice rendent en fait ces modèles inapplicables pour la plupart des économies modernes. Par exemple, en l'absence d'hypothèse d'efficacité des marchés, les avantages de la GEMVA ne se matérialisent pas nécessairement, et ce sont les firmes qui n'adoptent pas le modèle qui peuvent avoir de meilleures performances. De plus, dans certains cas particuliers comme par exemple les pays à croissance rapide tels que la Chine aujourd'hui, la préférence pour l'actionnaire ne s'accompagne pas généralement de performances élevées (Allen, 2005). On peut également craindre une incompatibilité de la préférence pour l'actionnaire avec des économies où les marchés sont liquides, ou les investisseurs poursuivent des objectifs de court terme et où les marchés financiers sont hautement instables (Aglietta et Rebérioux, 2005). On peut enfin observer qu' une structure de propriété très dispersée comme le système anglo-américain peut favoriser les scandales financiers, contrairement aux systèmes européens de propriété plus concentrés (Coffee, 2005). En conséquence, les bases de données ne doivent pas orienter l'interprétation du chercheur, mais doivent servir de support à leur analyse critique.

Pour ce qui est du second point, la prise en compte des disparités sectorielles permet de souligner le retour de la logique industrielle, oblitérée par la logique financière dans la gouvernance d'entreprise par la valeur actionnariale, et d'envisager une intégration des développements les plus récents en termes de dynamique ind ustrielle et de l'innovation. Nous avons comme résultat dans cet article que l'adoption de la GEMVA, tout en étant supérieure dans les TIC, rend les performances de cette industrie plus volatiles. Cela suggère que la GEMVA n'est pas nécessairement le meilleur modèle pour ce type d'industrie car il fragilise à terme la capacité à attirer des investisseurs qui recherchent des cours boursiers aux fluctuations limitées.

Les travaux sur la dynamique des structures de marché et sur les cycles de vie de l'industrie (Geroski, 1995 ; Audretsch et Lehmann, 2006 ; Klepper, 1997) reposent sur une dichotomie 
forte entre industries traditionnelles et industries innovatrices. Les conclusions en termes de gouvernance que l'on peut tirer de ces modèles est que les secteurs traditionnels peuvent être dominés par la GEMVA, alors que les secteurs innovateurs doivent bénéficier d'une gouvernance hybride où les actionnaires, les dirigeants et les autres parties prenantes peuvent intervenir conjointement dans la stratégie industrielle (Krafft et Ravix, 2007 ; Filatotchev et Wright, 2005).

En conclusion, nous pouvons indiquer quelques pistes de recherche futures dans cette ligne de discussion. Tout d'abord, le passage d'une évaluation monocritère à une évaluation multicritères peut permettre un progrès de l'analyse, mais il faut alors abandonner l'accumulation de critères hétéroclites au profit de critères complémentaires, articulés et pertinents. Par exemple, le fait que les pilules empoisonnées doivent être approuvées par les actionnaires (cf. Tableau 1) est contradictoire avec le principe selon lequel ces pratiques devraient être prohibées pour permettre le bon fonctionnement du « marché pour le contrôle des dirigeants » (Jensen, 1986). Un autre exemple concerne les rémunérations complémentaires comme les stocks options qui n'ont pas la même signification selon qu'elles sont offertes aux cadres dirigeants chargés d'exécuter la politique des actionnaires, ou bien à des chercheurs et à des collaborateurs dotés de ressources critiques dans l'objectif de les maintenir dans le processus de développement de l'entreprise (Lazonick et O'Sullivan, 2001).

Ensuite, la mise en évidence des disparités sectorielles dans les modes de gouvernance d'entreprise incite à explorer de manière plus systématique les liens entre dynamique industrielle et gouvernance d'entreprise. Ainsi, on peut penser que les stratégies de fusionacquisition ne sont pas une fin en soi. Elles ne sont pas un point d'arrivée, ni uniquement des moyens d'acquérir des compétences ou des capacités d'innovation. Les fusions acquisitions peuvent être aussi des moments dans le processus de cycle de vie de l'entreprise. Ces processus qui peuvent parfois conduire à des fusions, peuvent aussi impliquer des cessions. Dans ces conditions, une appréciation normative de la gouvernance d'entreprise qui serait fondée sur le rôle des OPA comme discipline des managers (Jensen, 1986) fait obstacle à une vision positive de l'OPA comme instrument de stratégie à confier aux dirigeants qui peuvent exercer leur capacité entrepreneuriale, alors que les financiers ne peuvent mesurer qu'a posteriori les résultats en termes de performance de marché ou de performance économique la gouvernance d'entreprise qu'ils confient aux dirigeants (Krafft et Ravix, 2008). 


\section{Notes}

${ }^{1}$ Ce travail fait partie d'un projet de recherche financé par l'Agence Nationale de la Recherche (références : ANR JCJC06_141306, «Knowledge intensive sectors : models and evidence »), la Région PACA, et le BQR de l'Université de Nice Sophia Antipolis. Il bénéficie aussi du soutien du CNRS au travers du centre de recherche Gredeg (UMR 6227).

2 Bebchuk et Weisbach (2009) montrent que le mot clef 'corporate governance' apparait dans le résumé de 987 articles recensés sur Social Science Research Network (SSRN) en 2008, ce qui a motivé la création du Corporate Governance Network (CGN) regroupant une vingtaine de journaux académiques publiant régulièrement sur ce thème.

${ }^{3}$ Le traitement des autres dimensions du tableau donnent des résultats équivalents à ceux que nous allons commenter. Une présentation complète de ces résultats donnera lieu à des publications ultérieures.

${ }^{4}$ Dans la base RM/ISS, le secteur agroalimentaire correspond aux produits alimentaires, aux boissons et au tabac.

5 Le secteur TIC correspond aux équipements de communication, aux ordinateurs et périphériques, aux équipements électroniques, à l'électronique de bureau, aux équipements et produits avec semi-conducteurs.

Tableau 1 : Mesures de bonne gouvernance et scores (source : RM/ISS)

\begin{tabular}{|c|c|c|}
\hline Dimensions & Mesu res de bonne gou vernance (GEMVA) & $\begin{array}{l}\text { Sous scores et scores } \\
\text { totaux }\end{array}$ \\
\hline \multicolumn{3}{|l|}{ Conseils } \\
\hline Composition du CA & Le CA doit être composé d'une large majorité de membres extérieurs & Sous score de 0 à 5 \\
\hline $\begin{array}{l}\text { Composition du comité de } \\
\text { gouvernance }\end{array}$ & Ce comité doit être composé d'une large majorité de membres extérieurs & Sous score de 0 à 5 \\
\hline $\begin{array}{l}\text { Composition du comité de } \\
\text { compensation }\end{array}$ & Ce comité doit être composé en intégralité de membres extérieurs & Sous score de 0 à 5 \\
\hline Taille et fréquence du CA & $\begin{array}{l}\text { Le CA doit comprendre entre } 6 \text { et } 15 \text { membres ( } 9 \text { à } 12 \text { étant considéré } \\
\text { comme idéal) et ses membres doivent rendre des comptes aux actionnaires } \\
\text { au moins une fois par an }\end{array}$ & Sous score de 0 à 5 \\
\hline $\begin{array}{l}\text { Changement dans lataille du } \\
\text { CA }\end{array}$ & $\begin{array}{l}\text { Les actionnaires doivent avoir le droit de voter sur les changements de taille } \\
\text { du CA }\end{array}$ & Sous score de 0 à 5 \\
\hline Vote cumulatifs & Les actionnaires doivent avoir le droit de cumuler leurs votes & Sous score de 0 à 5 \\
\hline $\begin{array}{l}\text { Participation du PDG à } \\
\text { d'autres CA }\end{array}$ & Le PDGne doit pas siéger dans plus de 2 autres CA & Sous score de 0 à 5 \\
\hline $\begin{array}{l}\text { Participation des dirigeants à } \\
\text { d'autres CA }\end{array}$ & Les autres membres du CA doivent être limités à 5 & Sous score de 0 à 5 \\
\hline $\begin{array}{l}\text { Participat ion d'anciens } \\
\text { dirigeants au CA }\end{array}$ & Les anciens PDG ne doivent pas siéger au CA & Sous score de 0 à 5 \\
\hline \multirow[t]{2}{*}{ PDG/Président du CA } & Les positions doivent être séparées & Sous score de 0 à 5 \\
\hline & & Total score de 0 à 50 \\
\hline \multicolumn{3}{|l|}{ Audit } \\
\hline $\begin{array}{l}\text { Composition du comité } \\
\text { d'audit }\end{array}$ & Ce comité doit être composé en intégralité de membres extérieurs & Sous score de 0 à 5 \\
\hline Changement d'auditeurs & La politique en matière de changement d'auditeur doit êtretransparente & Sous score de 0 à 5 \\
\hline \multirow[t]{2}{*}{ Ratification des auditeurs } & $\begin{array}{l}\text { Les actionnaires doivent pouvoir ratifier la sélection des auditeurs faite par } \\
\text { les dirigeants chaque année }\end{array}$ & Sous score de 0 à 5 \\
\hline & & Total score de $\mathbf{0}$ à 15 \\
\hline \multicolumn{3}{|l|}{ Charte/Statuts } \\
\hline Pilules empoisonnées & $\begin{array}{l}\text { Les actionnaires doivent pouvoir approuver les politiques en matière de } \\
\text { pilules empoisonnées }\end{array}$ & Sous score de 0 à 5 \\
\hline Vote & $\begin{array}{l}\text { Une majorité simple doit être adoptée pour amender les charte/stat uts et pour } \\
\text { approuver les fusions et coopérations }\end{array}$ & Sous score de 0 à 5 \\
\hline Consentement & Les actionnaires doivent pouvoir exprimer leur consentement par écrit & Sous score de 0 à 5 \\
\hline Réunions extraordinaires & Les actionnaires doivent pouvoir demander des réunions extraordinaires & Sous score de 0 à 5 \\
\hline
\end{tabular}




\begin{tabular}{|c|c|c|}
\hline $\begin{array}{l}\text { Amendements par les } \\
\text { dirigeants }\end{array}$ & $\begin{array}{l}\text { Les dirigeants ne doivent pas pouvoir amender les charte/stat uts sans } \\
\text { l'approbation des actionnaires }\end{array}$ & Sous score de 0 à 5 \\
\hline \multirow[t]{2}{*}{ Structure du capital } & Un vote doit correspondre à une action & Sous score de 0 à 5 \\
\hline & & Total score de 0 à 30 \\
\hline \multicolumn{3}{|l|}{$\begin{array}{l}\text { Compensation des dirigeants } \\
\text { et cadres }\end{array}$} \\
\hline $\begin{array}{l}\text { Approbation par les } \\
\text { actionnaires }\end{array}$ & $\begin{array}{l}\text { Tout plan d'incitation detype stock option doit être soumis à l'approbation } \\
\text { des actionnaires }\end{array}$ & Sous score de 0 à 5 \\
\hline \multirow[t]{2}{*}{ Compensation des dirigeants } & $\begin{array}{l}\text { Les dirigeants doivent recevoir une portion de leur compensation sous forme } \\
\text { d'actions }\end{array}$ & Sous score de 0 à 5 \\
\hline & & Total score de $\mathbf{0}$ à 10 \\
\hline \multicolumn{3}{|l|}{ Pratiques progressistes } \\
\hline $\begin{array}{l}\text { Limite aux mandats des } \\
\text { dirigeants }\end{array}$ & $\begin{array}{l}\text { Une limite aux mandats doit exister afin d'assurer le renouvellement régulier } \\
\text { du CA }\end{array}$ & Sous score de 0 à 5 \\
\hline Performance du CA & $\begin{array}{l}\text { Une étude de performance doit être menée et diffusée de manière } \\
\text { transparente }\end{array}$ & Sous score de 0 à 5 \\
\hline Plan de succession du PDG & $\begin{array}{l}\text { Le plan de succession doit être approuvé et évalué de manière régulière par } \\
\text { les actionnaires }\end{array}$ & Sous score de 0 à 5 \\
\hline \multirow[t]{3}{*}{ Conseillers extérieurs } & $\begin{array}{l}\text { La politique concernant la présence de conseillers extérieurs au conseil } \\
\text { d'administration doit être transparente }\end{array}$ & Sous score de 0 à 5 \\
\hline & & Total score de $\mathbf{0}$ à 20 \\
\hline & & $\begin{array}{l}\text { SCORE GLOBAL : } 0 \text { à } \\
125\end{array}$ \\
\hline
\end{tabular}

Tableau 2 : Statistiques descriptives Agroalimentaire versus TIC

\begin{tabular}{|l|c|c|c|c|c|c|}
\hline & & $\mathrm{N}$ & Mini & Maxi & Moyenne & Ecart-type \\
\hline \multirow{2}{*}{$\begin{array}{l}\text { Agro- } \\
\text { alimentaire }\end{array}$} & LnGEMVA & 42764 & -2.30 & 4.61 & 3.70 & 1.31 \\
\cline { 2 - 7 } & LnSP & 42729 & 0.01 & 10.52 & 4.52 & 1.88 \\
\hline \multirow{2}{*}{ TIC } & LnGEMVA & 28346 & 0.26 & 4.61 & 3.80 & 0.91 \\
\cline { 2 - 7 } & LnSP & 28352 & -0.87 & 7.32 & 3.67 & 1.57 \\
\hline
\end{tabular}

Tableau 3 : Corrélations entre LnGEMVA et LnSP avec modèle à effet fixe

\begin{tabular}{|c|c|c|c|c|}
\cline { 2 - 5 } \multicolumn{1}{c|}{} & \multicolumn{4}{c|}{ Coefficient on LnGEMVA } \\
\cline { 2 - 5 } \multicolumn{1}{c|}{} & $(1)$ & $(2)$ & $(3)$ & No.Obs \\
\hline Agro-alimentaire & $0.0791^{* * *}$ & $0.0083^{* * *}$ & $0.0142^{* * *}$ & 42646 \\
\hline TIC & $0.0367^{* * *}$ & $0.0333^{* * *}$ & $0.0701^{* * *}$ & 34081 \\
\hline Variables de contrôle & Aucune & MV & MV DY & \\
\hline *** Résultats significatifs à 1\% & & & \\
\hline
\end{tabular}




\section{Bibliographie}

Aglietta M. and Rébérioux A. (2005). Corporate Governance Adrift: a Critique of Shareholdervalue, Cheltenham: Edward Elgar.

Allen F. (2005). "Corporate governance in emerging economies", Oxford Review of Economic Policy, vol. 21(2), pp. 164-177.

Aoki M. (1984). The Cooperative Game Theory of the Firm, Oxford: Oxford University Press.

Audretsch D. and Lehmann E. (2006). "Entrepreneurial Access and Absorption of Knowledge Spillovers: Strategic Board and Managerial Composition for Competitive Advantage", Journal of Small Business Management, vol. 44(2), pp. 155-166.

Bebchuk L. and Hamdani, A. (2009). "The elusive quest for global governance standards", University of Pennsylvania Law Review, vol. 157(5), pp. 1263-1317.

Bebchuk L. and Weisbach M. (2009). "The state of corporate governance research", NBER WP 15537, forthcoming in the Review of Financial Studies.

Becht M., Jenkinson, T. and Mayer C. (2005). "Corporate governance: an assessment", Oxford Review of Economic Policy, vol. 21(2), pp. 155-163.

Blair M. (1995). Ownership and Control: Rethinking Corporate Governance for the Twenty First Century, Washington: Brookings Institution Press.

Coffee J. (2005). "A theory of corporate scandals: why the USA and Europe differ?", Oxford Review of Economic Policy, vol. 21(2), pp. 198-211.

Core J., Guay W. and Rusticus T. (2006). "Does weak governance cause weak stock returns? An examination of firm operating performance and investors' expectations", Journal of Finance, vol. 61(2), pp. 655-687.

Dasmodaran A. (2005). "Valuation approaches and metrics: a survey of the theory and evidence", Foundations and Trends in Finance, vol. 1(8), pp. 693-784.

Denis D. and McDonnel J. (2003). "International corporate governance", Journal of Financial and Quantitative Analysis, vol. 38, pp. 1-36.

Ertugrul M. and Hedge S. (2009). "Corporate governance ratings and firm performance", Financial Management, vol. 38, pp. 139-160.

Filatotchev I. and Wright M. (eds) (2005). The Life Cycle of Corporate Governance, Cheltenham: Edward Elgar.

Fransman M. (2004). "The telecoms boom and bust 1996-2003 and the role of financial markets", Journal of Evolutionary Economics, vol. 14(4), pp. 396-406.

Froud J., Haslam C., Johal S. and Williams K. (2000). "Shareholder value and financialization: consultancy promises, management moves", Economy and Society, vol. 29(1), pp. 80-110.

Geroski P. (1995). "What do we know about entry?", International Journal of Industrial Organization, vol. 13(4), pp. 421-440.

Gompers P., Ishii J. and Metrick A. (2003). "Corporate governance and equity prices", Quarterly Journal of Economics, vol. 118, pp. 107-155.

Grandori A. (Ed) (2004). Corporate Governance and Firm Organization - Microfoundations and Structural Forms, Oxford: Oxford University Press.

Hansmann H. (1996). The Ownership of Entreprise, Cambridge, MA: Harvard University Press.

Hart O. (1995). "Corporate governance: some theory and implications, Economic Journal, vol. 105(430), pp. 678-689. 
Jensen M. (1986). "Agency costs of free cash flow, corporate finance and takeovers", American Economic Review, vol. 76(2), pp. 323-329.

Jensen M. and Meckling W. (1976). "Theory of the firm: managerial behaviour agency costs and ownership structure", Journal of Financial Economics, vol. 3(4), pp. 305-360.

Klepper S. (1997). "Industry life cycles", Industrial and Corporate Change, vol. 6(1), pp. 119-143.

Koehn D. and Ueng, J. (2005). "Evaluating the evaluators : should investors trust corporate governance metrics ratings", Journal of Management and Governance, vol. 9, 111128.

Krafft J., Qu Y. and Ravix (2008). "Corporate governance, ind ustry dynamics and firms performance: an empirical analysis of a best practice model", Recherches Economiques de Louvain, vol. 74(4), pp. 455-478.

Krafft J. and Ravix J.L. (2008). "Corporate governance and the governance of knowledge: Rethinking the relationship in terms of corporate coherence", Economics of Innovation and New Technology, vol. 17(1-2), pp. 79-96.

Krafft J. and Ravix J. (2007). "The firm and its governance over the industry life cycle", Corporate Ownership and Control, vol. 5(1), pp. 233-242.

Krafft J. and Ravix J. (2005). "The governance of innovative firms : an evolutionary perspective", Economics of Innovation and New Technology, vol. 14(3), pp. 125-148.

Lazonick W. (2007). "The US stock market and the governance of innovative enterprise", Industrial and Corporate Change, vol. 16(6), pp. 983-1035.

Lazonick W. and O'Sullivan M. (2001). «Le rôle du marché boursier dans les systèmes nationaux de gouvernement d'entreprise », in M. Aglietta, Régimes de gouvernement d'entreprise, Rapport final pour le Commis sariat Général au Plan.

Lie E. (2005). "On the Timing of CEO Stock Option Awards", Management Science, vol. 51(5), pp. 802-812.

Shleifer A. and Vishny R. (1997). "A survey of corporate governance”, Journal of Finance, vol. 52, pp. 737-783.

Sonnenfeld J. (2004). "Good governance and the misleading myths of bad metrics", Academy of Management Executive, vol. 18(1), pp. 108-113.

Williamson O. (1988). "Corporate finance and corporate governance", Journal of Finance, vol. 43(3), pp. 567-591.

Zingales L. (2000). "In search for new foundations", Journal of Finance, vol. 55(4), pp. 16231653. 